\title{
Singular Homology and Class Field Theory of Varieties over Finite Fields
}

\author{
By Alexander Schmidt and Michael Spieß
}

Dedicated to the memory of Jürgen Neukirch

The aim of global class field theory is the description of abelian extensions of a finitely generated field $k$ in terms of arithmetic invariants attached to $k$. The solution of this problem in the case of fields of dimension 1 was one of the major achievements of number theory in the first part of the previous century.

In the 1980s, mainly due to K. Kato and S. Saito [KS2], a generalization to higher dimensional fields has been found. The description of the abelian extensions is given in terms of a generalized idèle class group, whose rather involved definition is based on Milnor $K$-sheaves. However, if one restricts attention to unramified extensions (with respect to a regular, projective model $\bar{X}$ of $k$ ), then class field theory has a nice geometric description using the Chow group $\mathrm{CH}_{0}(\bar{X})$ of zero-cycles modulo rational equivalence (see [KS1], [Sa]).

The objective of this paper is to give a description of a similar geometric flavor of the tamely ramified abelian extensions of $k$ (w.r.t. a finite set $Y_{1}, \ldots, Y_{n}$ of prime divisors of $\bar{X}$ ). We do this in the case of positive characteristic, where the role of $\mathrm{CH}_{0}(\bar{X})$ is taken over by the 0th singular homology group (introduced by A. Suslin) $h_{0}(X)$, where $X=\bar{X}-\left\{Y_{1} \cup \cdots \cup Y_{n}\right\}$. The arithmetic case, i.e. if $k$ is a finitely generated extension of $\mathbb{Q}$, will be considered in a separate paper.

Let $\bar{X}$ be a smooth, projective and geometrically connected variety over a finite field and let $X$ be an open subvariety of $\bar{X}$. Let $\pi^{t}(X)$ be the tame étale fundamental group of $X$ (cf. $[\mathrm{GM}]$ ), i.e. $\pi^{t}(X)$ classifies étale coverings of $X$ which are tamely ramified over all prime divisors of $\bar{X}$ not lying on $X$. Our main result is the existence of a natural map (reciprocity homomorphism)

$$
\text { rec }: h_{0}(X) \longrightarrow \pi_{1}^{t}(X)^{a b},
$$

which is injective with dense image and which induces an isomorphism on the profinite completions. Passing to small open subschemes, this completely de- 
scribes the tame part of the abelianized absolute Galois group of the function field of $X$.

Let us formulate the result in more detail. Sending a closed point $x$ of $X$ to its Frobenius in $\pi_{1}^{t}(X)^{a b}$ defines a homomorphism

$$
Z_{0}(X) \longrightarrow \pi_{1}^{t}(X)^{a b},
$$

where $Z_{0}(X)$ is the group of 0 -cycles on $X$. This map is known to have a dense image (see [L2] or [Ra], lemma 1.7), and we will see below that it factors through $h_{0}(X)$, which is a quotient of $Z_{0}(X)$ by definition.

The structural map $X \rightarrow \operatorname{Spec}(k)$ induces natural surjective homomorphisms

$$
\pi_{1}^{t}(X)^{a b} \rightarrow \pi_{1}(\operatorname{Spec}(k))^{a b} \cong \hat{\mathbb{Z}} \quad \text { and } \quad h_{0}(X) \stackrel{\operatorname{deg}}{\rightarrow} h_{0}(\operatorname{Spec}(k)) \cong \mathbb{Z}
$$

and we denote the kernels of these maps by $\left(\pi_{1}^{t}(X)^{a b}\right)^{0}$ and $h_{0}(X)^{0}$, respectively.

Theorem 0.1 The reciprocity homomorphism rec $: h_{0}(X) \rightarrow \pi_{1}^{t}(X)^{a b}$ induces an isomorphism

$$
\operatorname{rec}_{0}: h_{0}(X)^{0} \stackrel{\sim}{\longrightarrow}\left(\pi_{1}^{t}(X)^{a b}\right)^{0}
$$

of finite abelian groups which fits into the commutative diagram with exact rows

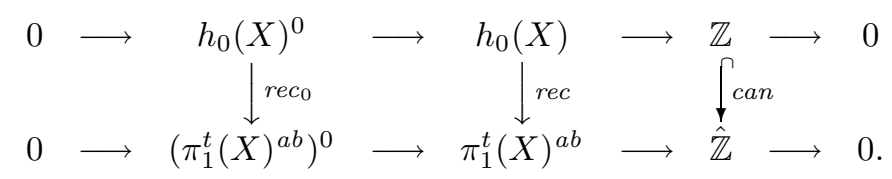

In particular, rec induces an isomorphism between the profinite completion of $h_{0}(X)$ and $\pi_{1}^{t}(X)^{a b}$.

Theorem 0.1 is well known in the following cases:

1. If $\operatorname{dim} X=1$, then (see [SV1], th.3.1) $h_{0}(X)$ is naturally isomorphic to the relative Picard group $\operatorname{Pic}(\bar{X}, \bar{X}-X)$. A straightforward computation identifies this relative Picard group with the ray class group of the function field $k(X)$ of $X$ with modulus $\mathfrak{m}_{X}$, where $\mathfrak{m}_{X}$ is the product of all primes of $\bar{X}-X$. In this case, rec is the reciprocity homomorphism of the classical (one-dimensional) class field theory for global fields of positive characteristic.

2. If $X$ is projective, then $h_{0}(X)$ coincides with the Chow group $\mathrm{CH}_{0}(X)$ of zero-dimensional cycles on $X$ modulo rational equivalence. In this case, theorem 0.1 is a reformulation of Kato's and Saito's unramified class field theory for $X$, see [KS1], [Sa] (and for two-dimensional $X$ we will eventually deduce theorem 0.1 from their result in the projective case). 
Let us make a remark on the role of the smooth compactification $\bar{X}$. First of all, $\pi_{1}^{t}(X)^{a b}$ does not depend on $\bar{X}$. Indeed, by the purity of the branch locus ([SGA1]), Exp.X, Th.3.1), we have a decomposition

$$
\pi_{1}^{t}(X)^{a b} \cong \pi_{1}(X)^{a b}(\text { prime-to- } p) \oplus \pi_{1}(\bar{X})^{a b}(p)
$$

and $\pi_{1}(\bar{X})$ is a birational invariant ([SGA1], Exp.X, Cor.3.4). We need $\bar{X}$ just for technical reasons. Its existence is known in dimension $\leq 2$ and any kind of desingularization theorem in positive characteristic would imply that theorem 0.1 holds for an arbitrary smooth, quasiprojective and geometrically connected variety over $k$.

In the arithmetic case i.e. when $X$ is a regular flat $\mathbb{Z}$-scheme of finite type one should expect a result similar to theorem 0.1. The first problem one encounters is that the singular homology groups are defined only for varieties over fields. However one can give an absolute version of singular homology for any scheme $X$ of finite type over $\mathbb{Z}$ (see [Sch] for their definitions and basic properties). The relation between the 0th absolute singular homology and the abelianized tame fundamental group will be investigated in a future work.

We want to thank D. Huybrechts for helpful e-mail conversation concerning the Bertini-type theorem 5.1.

\section{Notation and frequently used facts}

In this section, we collect some facts and notation from the papers [SV1], [SV2], [SV3], [V1], [V2] which will be frequently used in the subsequent sections. Recall Suslin's definition of the singular homology of varieties [SV1]. Let $k$ be a field which will always assumed to be perfect throughout this paper. Let $\Delta^{\bullet}$ be the standard cosimplicial object in the category of smooth schemes over $k$, i.e. $\Delta^{n}$ is the standard $n$-dimensional simplex given as a subscheme in $\mathbb{A}_{k}^{n+1}=$ $\operatorname{Spec}\left(k\left[T_{0}, \ldots, T_{n}\right]\right)$ by the equation $\Sigma T_{i}=1$ and the simplicial structure is given by the obvious face and degeneracy morphisms.

Let $X$ be a scheme of finite type over $k$. Denote by $C_{n}(X)$ the free abelian group generated by closed integral subschemes $Z \subset X \times \Delta^{n}$ such that the projection $Z \rightarrow \Delta^{n}$ is finite and surjective. One verifies immediately that if $Z$ is as above, then for each face map $\delta^{i}: \Delta^{n-1} \rightarrow \Delta^{n}$ each component of $\left(\delta^{i}\right)^{-1}(Z) \subset X \times \Delta^{n-1}$ is finite and surjective over $\Delta^{n-1}$ and hence has the "correct" dimension. So the cycle theoretic inverse image $\left(\delta^{i}\right)^{*}(Z)$ is well defined and lies in $C_{n-1}(X)$. This gives us face operators

$$
\partial_{i}=\left(\delta^{i}\right)^{*}: C_{n}(X) \longrightarrow C_{n-1}(X) .
$$

The homology groups of the complex

$$
(C \bullet(X), d), \quad d=\sum(-1)^{i} \delta_{i}
$$


will be denoted by $h_{i}(X)$ and are called the (integral) singular homology groups of $X$. For a positive integer $n$, the singular homology groups of $X$ with $\mathbb{Z} / n \mathbb{Z}$ coefficients are defined as the homology groups of the complex $C \bullet(X) \otimes \mathbb{Z} / n \mathbb{Z}$. An essential feature of these singular homology groups is that they naturally occur as homomorphism groups in Voevodsky's "triangulated category of motivic complexes" $\mathrm{DM}_{-}^{\mathrm{eff}}(k)$. As in [V1], we consider the following categories:

$$
\begin{array}{ll}
\operatorname{Sm}(k) & \text { - the category of smooth schemes over } \operatorname{Spec}(k) \\
& \text { with scheme morphisms, } \\
\operatorname{SmCor}(k) & \text { the category of smooth schemes over } \operatorname{Spec}(k) \\
& \text { with finite correspondences as morphisms, } \\
\operatorname{Shv}_{\mathrm{Nis}}(\operatorname{SmCor}(k)) \quad \begin{array}{l}
\text { - the category of Nisnevich sheaves of } \\
\text { abelian groups on } \operatorname{SmCor}(k) .
\end{array}
\end{array}
$$

We refer to presheaves on $\operatorname{SmCor}(k)$ also as presheaves with transfer on $\operatorname{Sm}(k)$. A presheaf $F$ on $\operatorname{Sm}(k)$ is called homotopy invariant if for every $U \in \operatorname{Sm}(k)$ the natural projection $p: U \times \mathbb{A}_{k}^{1} \rightarrow U$ induces an isomorphism $F(U) \stackrel{\sim}{\longrightarrow} F\left(U \times \mathbb{A}_{k}^{1}\right)$. If $F$ is a homotopy invariant presheaf with transfers, then also the sheafification $F_{\text {Nis }}$ is homotopy invariant (see [V1], th.3.1.11). We use the following notation.

$$
\begin{aligned}
\mathrm{D}^{-}\left(\operatorname{Shv}_{\mathrm{Nis}}(\operatorname{SmCor}(k))\right) \quad & \text { the derived category of bounded above } \\
& \text { complexes in } \operatorname{Shv}_{\mathrm{Nis}}(\operatorname{SmCor}(k)), \\
\mathrm{DM}_{-}^{\mathrm{eff}}(k) & - \text { the full subcategory of } \mathrm{D}^{-}\left(\operatorname{Shv}_{\mathrm{Nis}}(\operatorname{SmCor}(k))\right) \\
& \text { consisting of complexes with homotopy } \\
& \text { invariant cohomology sheaves. }
\end{aligned}
$$

$\mathrm{DM}_{-}^{\mathrm{eff}}(k)$ is a triangulated category in a natural way. For a presheaf $F$ on $\operatorname{Sm}(k)$, we consider the complex $\underline{C}$. $(F)$ of presheaves on $\operatorname{Sm}(k)$ with $\underline{C}_{n}(F)(X)=$ $F\left(\Delta^{n} \times X\right)$ and with differentials given by the alternating sum of the morphisms which correspond to the boundary morphisms of $\Delta^{\bullet}$. We denote the homology presheaves of $\underline{C}$. $(F)$ by $\underline{h}_{i}(F)$. We set $C_{\bullet}(F)=\underline{C}$. $(F)(\operatorname{Spec}(k))$ and $h_{i}(F)=$ $\underline{h}_{i}(F)(\operatorname{Spec}(k))$. Further, $\underline{h}_{i}^{\mathrm{Nis}}(F)$ denotes the homology sheaves of $\underline{C}$. $(F)_{\text {Nis }}$ and we write $h_{i}^{\mathrm{Nis}}(F)$ for $\underline{h}_{i}^{\mathrm{Nis}}(F)(\operatorname{Spec}(k))$. Note that $\underline{C}$. $(F)$ is a complex of sheaves if $F$ is a sheaf, but in general $\underline{C} .\left(F_{\mathrm{Nis}}\right)$ and $\underline{C} .(F)_{\text {Nis }}$ differ. If $F$ is a presheaf on $\operatorname{SmCor}(k)$, then the sheaves $\underline{h}_{i}^{\text {Nis }}(F)$ are homotopy invariant (see [V1], th.3.1.11). This yields a functor

$$
\underline{C} .: \operatorname{Shv}_{\mathrm{Nis}}(\operatorname{SmCor}(k)) \rightarrow \mathrm{DM}_{-}^{\mathrm{eff}}(k) .
$$

Suppose we are given schemes $X, Y$ of finite type over $k$ and an integer $r \geq 0$. We consider the presheaves $z_{0}(X)$ and $c_{0}(X)$ on $\operatorname{SmCor}(k)$ which are given for $U \in \operatorname{Sm}(k)$ by

$$
\begin{array}{ll}
z_{0}(X)(U) \quad \text { - the free abelian group over cycles } Z \subset X \times U, \\
\text { quasifinite and dominant over an irreducible component of } U \\
c_{0}(X)(U) \begin{aligned}
\text { - the free abelian group over cycles } Z \subset X \times U, \text { finite } \\
\text { and surjective over an irreducible component of } U .
\end{aligned}
\end{array}
$$


These presheaves are Nisnevich sheaves (even qfh-sheaves, see [SV2], th.4.2.9). If $X$ is smooth, then $c_{0}(X)(U)=\operatorname{Hom}_{\operatorname{SmCor}(k)}(U, X)$. The complex $\underline{C} \cdot\left(c_{0}(X)\right)$ defines an object $M(X)$ in $\mathrm{DM}_{-}^{\mathrm{eff}}(k)$ which is called the motivic complex attached to $X$. For an integer $n$, we use the notation $M(X, \mathbb{Z} / n \mathbb{Z})$ for the object in $\mathrm{DM}_{-}^{\mathrm{eff}}(k)$ which is given by the complex $\underline{C} .\left(c_{0}(X) \otimes \mathbb{Z} / n \mathbb{Z}\right) . M(X)$ is covariant functorial with respect to scheme morphisms and for smooth schemes with respect to finite correspondences. We write $\mathbb{Z}$ for $M(\operatorname{Spec}(k))$.

By ([V1], prop.3.2.3), $\underline{C}$. naturally extends to a functor

$$
\mathbf{R} \underline{C}: \mathrm{D}^{-}\left(\operatorname{Shv}_{\mathrm{Nis}}(\operatorname{SmCor}(k))\right) \longrightarrow \mathrm{DM}_{-}^{\mathrm{eff}}(k),
$$

and (loc. cit.) one can give an alternative description of $\mathrm{DM}_{-}^{\text {eff }}(k)$ as the localization of $\mathrm{D}^{-}\left(\operatorname{Shv}_{\mathrm{Nis}}(\mathrm{SmCor}(k))\right)$ with respect to the localizing subcategory generated by complexes of the form $c_{0}\left(X \times \mathbb{A}_{k}^{1}\right) \stackrel{c_{0}(p r)}{\longrightarrow} c_{0}(X)$ for smooth schemes $X$ over $k$.

For a smooth scheme $X$, we have (see [V1], th.3.2.6) a natural isomorphism in $\mathrm{DM}_{-}^{\mathrm{eff}}(k)$

$$
\underline{C} \cdot\left(c_{0}(X)\right) \stackrel{\sim}{\longrightarrow} \underline{C}\left(c_{0}(X)\right) .
$$

If $X$ and $Y$ are smooth, then (see [V1], cor.3.2.7)

$$
\operatorname{Hom}_{\mathrm{DM}_{-}^{\mathrm{eff}}(k)}(M(X), M(Y)[n])=\mathbf{H}_{\mathrm{Nis}}^{n}\left(X, \underline{C} .\left(c_{0}(Y)\right)\right) .
$$

In particular, for smooth $X$

$$
\operatorname{Hom}_{\mathrm{DM}_{-}^{\mathrm{eff}}(k)}(\mathbb{Z}[n], M(X))=h_{n}^{\mathrm{Nis}}\left(c_{0}(X)\right)=h_{n}\left(c_{0}(X)\right)=h_{n}(X) .
$$

Using left resolutions by presheaves with transfers of the form $c_{0}(X)$ with $X$ smooth, and using the characterization of $\mathrm{DM}_{-}^{\text {eff }}(k)$ as a localization of the category $\mathrm{D}^{-}\left(\operatorname{Shv}_{\mathrm{Nis}}(\operatorname{SmCor}(k))\right)$, one constructs a tensor product on $\mathrm{DM}_{-}^{\mathrm{eff}}(k)$ ([V1], §3).

Recall the definition of the Tate object $\mathbb{Z}(1)$ in $\mathrm{DM}_{-}^{\text {eff }}(k)$ (cf. [SV3], §3). Let $\mathbb{G}_{m}$ denote the multiplicative group scheme $\mathbb{A}_{k}^{1}-\{0\}$. Let, for $i=1, \cdots, n$, $D_{i}\left(\mathbb{G}_{m}^{\times(n-1)}\right)$ be the direct summand in $c_{0}\left(\mathbb{G}_{m}^{\times n}\right)$ which is given by the homomorphism

$$
c_{0}\left(\mathbb{G}_{m}^{\times(n-1)}\right) \longrightarrow c_{0}\left(\mathbb{G}_{m}^{\times n}\right), \quad\left(x_{1}, \ldots, x_{n-1}\right) \mapsto\left(x_{1}, \ldots, 1_{i}, \ldots, x_{n-1}\right)
$$

We consider the sheaf $c_{0}\left(\mathbb{G}_{m}^{\wedge n}\right)$ which is defined as the direct summand of $c_{0}\left(\mathbb{G}_{m}^{\times n}\right)$ complementary to the direct summand $\sum_{i=1}^{n} D_{i}\left(G_{m}^{\times(n-1)}\right)$. The Tate object $\mathbb{Z}(n)$ is defined as $C \cdot\left(c_{0}\left(\mathbb{G}_{m}^{\wedge n}\right)\right)[-n]$. There are natural quasiisomorphisms $\mathbb{Z}(n) \otimes \mathbb{Z}(m) \stackrel{\sim}{\longrightarrow} \mathbb{Z}(n+m)$ for $n, m \geq 0$. For an arbitrary $M \in \mathrm{DM}_{-}^{\text {eff }}(k)$, one defines

$$
M(n)=M \otimes \mathbb{Z}(n)
$$


The complex $\mathbb{Z}(n)$ is acyclic in (cohomological) degrees $>n$, thus for an étale $k$-scheme $Z$, we have

$$
\operatorname{Hom}_{\mathrm{DM}_{-}^{\mathrm{eff}}(k)}(\mathbb{Z}, M(Z)(q)[p])=0 \quad \text { for } p>q .
$$

Furthermore, by ([SV3], th.3.4), we have a natural isomorphism

$$
\operatorname{Hom}_{\mathrm{DM}_{-}^{\text {eff }}(k)}(\mathbb{Z}, M(Z)(2)[2]) \cong K_{2}(Z) .
$$

Since $K_{2}$ of finite fields vanishes, we obtain the

Proposition 1.1 If $k$ is finite and if $Z$ is an étale $k$-scheme, then

$$
\operatorname{Hom}_{\mathrm{DM}_{-}^{\text {eff }}(k)}(\mathbb{Z}, M(Z)(2)[i])=0 \quad \text { for } i \geq 2
$$

and the same is true with $M(Z)$ replaced by $M(Z, \mathbb{Z} / n \mathbb{Z})$ for an arbitrary positive integer $n$.

Next we recall some well known facts on the higher Chow groups defined by Bloch [B1]. Let $X$ be an equidimensional scheme of finite type over $k$. Let $z^{r}(X, n)$ be the free abelian group generated by codimension $r$ subvarieties $V \subset$ $X \times \Delta^{n}$ which intersect all faces $X \times \Delta^{m}, m<n$ properly (i.e. every irreducible component of the intersection has the right dimension). Using the alternating sum of the homomorphisms associated to the face morphisms, one obtains a complex $z^{r}(X, \bullet)$ in the usual way, and the higher Chow groups (respectively the higher Chow groups with $\mathbb{Z} / n \mathbb{Z}$-coefficients) are defined by

$$
\begin{gathered}
\mathrm{CH}^{r}(X, i):=H^{-i}\left(z^{r}(X, \bullet)\right) \quad \text { and } \\
\mathrm{CH}^{r}(X, i, \mathbb{Z} / n \mathbb{Z}):=H^{-i}\left(z^{r}(X, \bullet, \mathbb{Z} / n \mathbb{Z})\right),
\end{gathered}
$$

respectively (where $\left.z^{r}(X, \bullet, \mathbb{Z} / n \mathbb{Z})=z^{r}(X, \bullet) \otimes \mathbb{Z} / n \mathbb{Z}\right)$. Immediately from the definition, we have

$$
\mathrm{CH}^{r}(X, i)=0 \quad \text { for } r>\operatorname{dim} X+i .
$$

The higher Chow groups are covariant functorial for proper maps and contravariant for flat map (for arbitrary maps, if $X$ is smooth and affine). The higher Chow sheaves

$$
\mathbf{C H}^{d}(X, i)
$$

are defined as the sheaves on $X_{\text {Zar }}$ associated to $U \mapsto \mathrm{CH}^{d}(U, i)$. By ([B1], th.3.2), there exists a local to global spectral sequence

$$
E_{2}^{p q}=H_{\mathrm{Zar}}^{p}\left(X, \mathbf{C H}^{r}(X,-q)\right) \Longrightarrow \mathrm{CH}^{r}(X,-p-q) .
$$

Let $X$ be a smooth variety and denote by $X^{j}$ the set of points of codimension $j$ on $X$. There are flasque resolutions of Zariski sheaves for all $r$ (Gersten resolutions; see [B1], th.10.1) 


$$
\begin{array}{r}
\left.\left.\mathbf{C H}^{r}(X, q) \hookrightarrow \bigoplus_{x \in X^{0}} i_{x *} \mathrm{CH}^{r}(\operatorname{Spec}(k(x)), q)\right) \rightarrow \bigoplus_{x \in X^{1}} i_{x *} \mathrm{CH}^{r-1}(\operatorname{Spec}(k(x)), q-1)\right) \\
\left.\rightarrow \cdots \rightarrow \bigoplus_{x \in X^{q}} i_{x *} \mathrm{CH}^{r-q}(\operatorname{Spec}(k(x)), 0)\right) \rightarrow 0,
\end{array}
$$

and a similar result holds for $\mathbb{Z} / n \mathbb{Z}$-coefficients as well.

Furthermore, we have the following connection to Milnor $K$-theory (see [NS], th.4.9 or $[\mathrm{To}])$ : For any field $k$

$$
\mathrm{CH}^{r}(\operatorname{Spec}(k), r) \cong K_{r}^{M}(k) .
$$

From the partial degeneration of the local to global spectral sequence, one obtains the

Proposition 1.2 For $X$ smooth and quasiprojective, we have isomorphisms

$$
\mathrm{CH}^{p}(X, i) \cong H_{\mathrm{Zar}}^{p-i}\left(X, \mathbf{C H}^{p}(X, p)\right) \quad \text { for } 0 \leq i \leq 2 .
$$

and the same holds for $\mathbb{Z} / n \mathbb{Z}$-coefficients as well.

The theorem of Suslin/Nesterenko/Totaro together with the Gersten resolution for $\mathbb{Z} / n \mathbb{Z}$-coefficients imply the

Proposition 1.3 If $X$ is smooth, quasiprojective of dimension $d$, then there exists a flasque resolution

$$
\begin{array}{r}
0 \rightarrow \mathbf{C H}^{d}(X, d, \mathbb{Z} / n \mathbb{Z}) \rightarrow \bigoplus_{x \in X^{0}} i_{x *}\left(K_{d}^{M}(k(x)) / n\right) \rightarrow \bigoplus_{x \in X^{1}} i_{x *}\left(K_{d-1}^{M}(k(x)) / n\right) \\
\left.\rightarrow \cdots \rightarrow \bigoplus_{x \in X^{d}} i_{x *}\left(K_{0}^{M}(k(x)) / n\right)\right) \rightarrow 0 .
\end{array}
$$

\section{Comparison results}

Many of the deeper theorems on algebraic cycle complexes depend on the fact that if $F$ is a presheaf on $\operatorname{Sm}(k)$ satisfying the property $(*)$ below, then $\underline{h}_{i}^{\mathrm{Nis}}(F)=0$ for all $i$. This is proved in ([FV], th. 5.5.2) under the assumption that the field $k$ admits resolution of singularities (ROS, for short) in the sense of ([FV], definition 3.4). Following the arguments in [V2], [FV], we prove a weaker result, which, however, only depends on ROS for schemes of dimension $\leq 2$ (which is known).

For a presheaf $F$ on $\operatorname{Sm}(k)$, we consider the following property $(*)$ :

"For any smooth scheme $X$ over $k$ and any $x \in F(X)$ there exists a proper birational morphism $p: X^{\prime} \rightarrow X$ such that $p^{*}(x)=0 . "$

By $(*) \leq 2$ we will refer to the property that the statement of $(*)$ holds for all smooth schemes over $k$ of dimension $\leq 2$. For a smooth scheme $X$, we denote by $\mathbb{Z}(X)$ the presheaf of abelian groups on $\operatorname{Sm}(k)$ freely generated by the presheaf of sets represented by $X$. We need the following results. 
Proposition 2.1 Let $k$ be a perfect field, let $X$ be a smooth scheme over $k$ and let $Z \subset X$ be a smooth closed subscheme. Denote by $p: X_{Z} \rightarrow X$ the blow-up of $X$ with center in $Z$. Then, for every homotopy invariant $G \in \operatorname{Shv}_{\mathrm{Nis}}(\operatorname{SmCor}(k))$ and any $i$, one has

$$
\operatorname{Ext}_{\operatorname{Shv}_{\mathrm{Nis}}(\operatorname{Sm}(k))}^{i}\left(\operatorname{coker}\left(\mathbb{Z}\left(X_{Z}\right) \stackrel{\mathbb{Z}(p)}{\rightarrow} \mathbb{Z}(X)\right)_{\mathrm{Nis}}, G\right)=0 .
$$

Proof: This follows from ([V2], prop.5.21) and ([V1], prop.3.1.10).

Lemma 2.2 Let $F$ be a presheaf on $\operatorname{Sm}(k)$ and let $G \in \operatorname{Shv}_{\mathrm{Nis}}(\operatorname{SmCor}(k))$ be homotopy invariant. Then for all $i$

$$
\operatorname{Hom}_{\mathrm{D}^{-}\left(\operatorname{Shv}_{\mathrm{Nis}}(\operatorname{Sm}(k))\right)}\left(\underline{C} \cdot(F)_{\mathrm{Nis}}, G[i]\right) \cong \operatorname{Ext}_{\operatorname{Shv}_{\mathrm{Nis}}(\operatorname{Sm}(k))}^{i}(F, G) .
$$

Proof: See ([FV], lemma 5.6) resp. the proof of ([V2], th.5.9).

Corollary 2.3 Let $p: X_{Z} \rightarrow X$ be as in proposition 2.1. Then

$$
\underline{h}_{i}^{\mathrm{Nis}}\left(\operatorname{coker}\left(\mathbb{Z}\left(X_{Z}\right) \stackrel{\mathbb{Z}(p)}{\rightarrow} \mathbb{Z}(X)\right)\right)=0 \quad \text { for all } i .
$$

Proof: (cf. the last part of the proof of [FV], th.5.5). Put $F=\operatorname{coker}\left(\mathbb{Z}\left(X_{Z}\right) \rightarrow\right.$ $\mathbb{Z}(X))$. Suppose $\underline{h}_{n}^{\mathrm{Nis}}(F) \neq 0$ for some $n$. We may assume that $\underline{h}_{i}^{\mathrm{Nis}}(F)=0$ for all $i<n$. Then there is a nontrivial morphism in $\mathrm{D}^{-}\left(\operatorname{Shv}_{\mathrm{Nis}}(\operatorname{Sm}(k))\right)$ of the form $\underline{C}_{\boldsymbol{}}(F)_{\mathrm{Nis}} \rightarrow \underline{h}_{n}^{\mathrm{Nis}}(F)[n]$. Therefore the statement follows from 2.2 and 2.1

Now we are prepared to prove the

Theorem 2.4 Let $F$ be a presheaf on $\operatorname{Sm}(k)$ satisfying the property $(*) \leq 2$. Then

$$
h_{i}^{\mathrm{Nis}}(F)=0 \quad \text { for } i \leq 2 .
$$

Proof: A proper birational morphism of smooth schemes of dimension $\leq 1$ is an isomorphism, thus $F(U)=0$ for any smooth $U$ of dimension $\leq 1$. In particular,

$$
\underline{C}_{i}(F)_{\mathrm{Nis}}(\operatorname{Spec}(k))=0 \quad \text { for } i=0,1,
$$

which shows the assertion for $i=0,1$.

In order to deal with the case $i=2$, we first consider the special case that $F$ is of the form

$$
\operatorname{coker}\left(\mathbb{Z}\left(X^{\prime}\right) \stackrel{\mathbb{Z}(p)}{\longrightarrow} \mathbb{Z}(X)\right)
$$


where $p: X^{\prime} \longrightarrow X$ is a birational morphism of smooth $k$-schemes of dimension 2 which is a composition of $n$ blow-ups with smooth centers (presheaves of this type satisfy property $(*))$. We proceed by induction on $n$ as in the proof of ([FV], lemma 5.4). Let $p=p_{0} \circ p_{1}$, where $p_{0}$ is a blow-up with a smooth center and $p_{1}$ is a composition of $n-1$ blow-ups with smooth centers. By induction on $n$ and corollary 2.3 , we may assume that $h_{i}^{\mathrm{Nis}}\left(\operatorname{coker}\left(\mathbb{Z}\left(p_{\epsilon}\right)\right)\right)=0$ for $i \leq 2$ and $\epsilon=0,1$. We have an exact sequence of presheaves on $\operatorname{Sm}(k)$

$$
0 \rightarrow \Psi \rightarrow \operatorname{coker}\left(\mathbb{Z}\left(p_{1}\right)\right) \rightarrow \operatorname{coker}(\mathbb{Z}(p)) \rightarrow \operatorname{coker}\left(\mathbb{Z}\left(p_{0}\right)\right) \rightarrow 0 .
$$

We rewrite this as two short exact sequences

$$
\begin{array}{r}
0 \rightarrow \Psi \rightarrow \operatorname{coker}\left(\mathbb{Z}\left(p_{1}\right)\right) \rightarrow \Psi^{\prime} \rightarrow 0, \\
0 \rightarrow \Psi^{\prime} \rightarrow \operatorname{coker}(\mathbb{Z}(p)) \rightarrow \operatorname{coker}\left(\mathbb{Z}\left(p_{0}\right)\right) \rightarrow 0 .
\end{array}
$$

As a subpresheaf of $\operatorname{coker}\left(\mathbb{Z}\left(p_{1}\right)\right.$ ) (resp. of $\operatorname{coker}(\mathbb{Z}(p))$ ), the presheaf $\Psi$ (resp. $\left.\Psi^{\prime}\right)$ satisfies property $(*)$. Thus $h_{i}^{\mathrm{Nis}}(\Psi)=0=h_{i}^{\mathrm{Nis}}\left(\Psi^{\prime}\right)$ for $i=0,1$ and the long exact $h_{i}^{\mathrm{Nis}}$-sequences associated with the two short exact sequences above imply that

$$
h_{i}^{\mathrm{Nis}}(\operatorname{coker}(\mathbb{Z}(p)))=0 \quad \text { for } i \leq 2 .
$$

For a general presheaf $F$ on $\operatorname{Sm}(k)$ satisfying property $(*) \leq 2$, we consider the homomorphism

$$
\bigoplus \phi_{\alpha}: \bigoplus \mathbb{Z}\left(X_{\alpha}\right) \longrightarrow F
$$

where the sum is taken over all pairs of the form $\left(X_{\alpha}, \phi_{\alpha}\right)$, where $X_{\alpha}$ is a smooth scheme of dimension 2 over $k$ and $\phi_{\alpha} \in F\left(X_{\alpha}\right)$. It follows from property $(*) \leq 2$ and from the resolution of singularities for schemes of dimension $2[\mathrm{Li}]$ that the above homomorphism may by factored through a homomorphism of the form

$$
\bigoplus \operatorname{coker}\left(\mathbb{Z}\left(X^{\prime}\right) \stackrel{\mathbb{Z}(p)}{\rightarrow} \mathbb{Z}(X)\right) \longrightarrow F
$$

where each of the morphisms $p$ is a birational morphism between smooth $k$ schemes of dimension 2 which a composition of finitely many blow-ups with smooth centers and such that the cokernel $\Psi_{2}$ of (2) has the property $\Psi_{2}(U)=0$ for every smooth $k$-scheme $U$ of dimension $\leq 2$. Denote by $\Psi_{0}$ and $\Psi_{1}$ the kernel and the image of the homomorphism (2). Thus we have short exact sequences of presheaves on $\operatorname{Sm}(k)$

$$
\begin{array}{r}
0 \rightarrow \Psi_{0} \rightarrow \bigoplus \operatorname{coker}\left(\mathbb{Z}\left(X^{\prime}\right) \stackrel{\mathbb{Z}(p)}{\longrightarrow} \mathbb{Z}(X)\right) \rightarrow \Psi_{1} \rightarrow 0 \\
0 \longrightarrow \Psi_{1} \longrightarrow F \longrightarrow \Psi_{2} \longrightarrow 0
\end{array}
$$

Each of the presheaves $\operatorname{coker}\left(\mathbb{Z}\left(X^{\prime}\right) \stackrel{\mathbb{Z}(p)}{\rightarrow} \mathbb{Z}(X)\right)$ satisfies property $(*)$, and therefore also $\Psi_{0}$ has this property. Furthermore, as a subpresheaf of $F$, also $\Psi_{1}$ satisfies property $(*) \leq 2$. Thus

$$
h_{i}^{\mathrm{Nis}}\left(\Psi_{0}\right)=h_{i}^{\mathrm{Nis}}\left(\Psi_{1}\right)=0 \quad \text { for } i=0,1 .
$$


Since $\Psi_{2}(X)=0$ for every smooth scheme of dimension $\leq 2$, we obtain

$$
h_{i}^{\mathrm{Nis}}\left(\Psi_{2}\right)=0 \quad \text { for } i \leq 2 .
$$

The long exact sequences associated to (3) and (4) together with (1) therefore yield the assertion.

Let us recall the following results.

Theorem 2.5 (Suslin, [S]) Let $X$ be an affine, equidimensional scheme of finite type over $k$ of dimension $d$. Then the natural inclusion of complexes of abelian groups

$$
C \bullet\left(z_{0}(X)\right) \longrightarrow z^{d}(X, \bullet)
$$

is a quasiisomorphism.

Theorem 2.6 (Suslin-Voevodsky, [SV2] th.4.3.1) Let $X$ be a scheme of finite type over $k$, let $Y$ be a closed subscheme of $X$ and let $j: U \rightarrow X$ be the open complement of $Y$ in $X$. Then the sequence of presheaves on $\operatorname{SmCor}(k)$

$$
0 \longrightarrow z_{0}(Y) \longrightarrow z_{0}(X) \stackrel{j^{*}}{\longrightarrow} z_{0}(U)
$$

is exact and coker $\left(j^{*}\right)$ satisfies property $(*)$.

Now we are in the position to prove the

Theorem 2.7 Let $X$ be a smooth, projective scheme of dimension $d \leq 2$ over a perfect field $k$. Then the canonical map

$$
h_{i}(X) \longrightarrow C H^{d}(X, i)
$$

is an isomorphism for $i=0,1$. The same result holds for $\mathbb{Z} / n \mathbb{Z}$-coefficients.

Proof: The case $d=0$ is trivial. We proceed by induction on $d$. Assume that $d \geq 1$ and that the theorem is true in dimension $<d$.

Choose a smooth hyperplane section $H \subset X$ and let $Y=X-H$ (observe that $Y$ is affine). By 2.6, we have an exact sequence of presheaves on $\operatorname{SmCor}(k)$

$$
0 \longrightarrow c_{0}(H) \longrightarrow c_{0}(X) \stackrel{j^{*}}{\longrightarrow} z_{0}(Y)
$$

and $F:=\operatorname{coker}\left(j^{*}\right)$ satisfies property $(*)$. We split (5) into two short exact sequences

$$
\begin{array}{r}
0 \longrightarrow c_{0}(H) \longrightarrow c_{0}(X) \longrightarrow G \longrightarrow 0 \\
0 \longrightarrow G \longrightarrow z_{0}(Y) \longrightarrow F \longrightarrow 0 .
\end{array}
$$


Consider the following diagram with exact columns (the right column is the localization sequence for higher Chow groups, [B2])

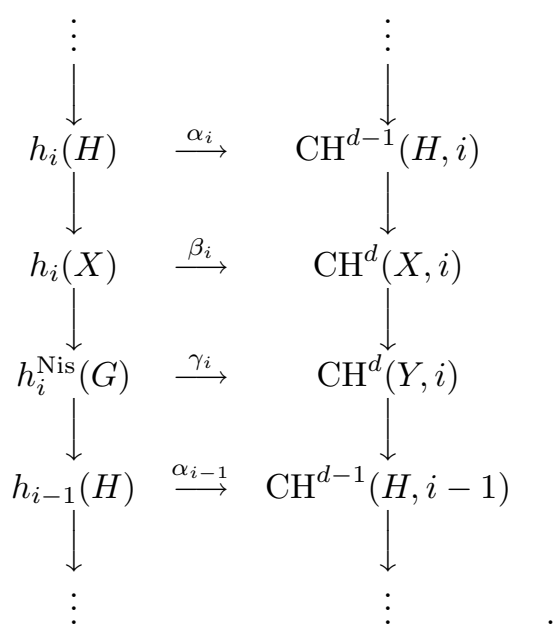

The map $\gamma_{i}$ is the composition

$$
h_{i}^{\mathrm{Nis}}(G) \longrightarrow h_{i}^{\mathrm{Nis}}\left(z_{0}(Y)\right)=h_{i}\left(z_{0}(Y)\right) \stackrel{\sim}{\longrightarrow} C H^{d}(Y, i) .
$$

The right hand isomorphism follows from 2.5, since $Y$ is affine. By 2.4, $\gamma_{i}$ is an isomorphism for $i=0,1$ and surjective for $i=2$. Therefore the induction step follows from the five-lemma.

If the field $k$ is finite, then the existence of a smooth hyperplane section $H$ is only granted over a finite extension field of $k$ whose degree, however, can be chosen prime to an arbitrary given integer. Considering two hyperplane sections $H_{1}, H_{2}$ defined over finite extensions $k_{1}, k_{2}$ of $k$ with coprime degree, we conclude the induction step by the usual restriction-corestriction argument.

Corollary 2.8 Let $C$ be a smooth, projective curve over $k$. Then we have a natural isomorphism

$$
\operatorname{Hom}_{\mathrm{DM}_{-}^{\text {eff }}(k)}(\mathbb{Z}, M(C)(1)[1]) \cong \mathrm{CH}^{2}(C, 1) .
$$

If the field $k$ is finite, then both groups are finite of order prime to char $(k)$.

Furthermore,

$$
\operatorname{Hom}_{\mathrm{DM}_{-}^{\text {eff }}(k)}(\mathbb{Z}, M(C)(1)[2])=0 .
$$

The same result holds for $\mathbb{Z} / n \mathbb{Z}$-coefficients.

Proof: For the surface $C \times \mathbb{P}^{1}$, we have the following decompositions for the 
Suslin homology ([V1], prop.3.5.1) and for the higher Chow groups ([B1], th.7.1)

$$
\begin{array}{ccccc}
h_{1}\left(C \times \mathbb{P}^{1}\right) & \cong & h_{1}(C) & \oplus & \operatorname{Hom}_{\mathrm{DM}_{-}^{\text {eff }}(k)}(\mathbb{Z}, M(C)(1)[1]), \\
C H^{2}\left(C \times \mathbb{P}^{1}, 1\right) & \cong C H^{1}(C, 1) & \oplus & C H^{2}(C, 1) .
\end{array}
$$

By construction, the natural homomorphism $h_{1}\left(C \times \mathbb{P}^{1}\right) \rightarrow C H^{2}\left(C \times \mathbb{P}^{1}, 1\right)$ respects these decompositions, hence the first isomorphism of the corollary follows from 2.7. By 1.2, we have an isomorphism

$$
\mathrm{CH}^{2}(C, 1) \cong H_{\mathrm{Zar}}^{1}\left(C, \mathbf{C H}^{2}(C, 2)\right) .
$$

The Gersten resolution for $\mathbf{C} \mathbf{H}^{2}(C, 2)$ implies that

$$
\mathrm{CH}^{2}(C, 1) \cong \operatorname{coker}\left(K_{2}(k(C)) \longrightarrow \bigoplus_{x \in C^{1}} k(x)^{*}\right)
$$

If $k$ is finite, a theorem of C. Moore ([Mo], th.7.4) shows that this cokernel is isomorphic to the product of the multiplicative groups of the fields of constants of the function fields of the connected components of $C$. In particular, it is finite of order prime to $p$.

In the same manner as above, we obtain natural decompositions

$$
\begin{array}{ccccc}
h_{0}\left(C \times \mathbb{P}^{1}\right) & \cong & h_{0}(C) & \oplus & \operatorname{Hom}_{\mathrm{DM}_{-}^{\text {eff }}(k)}(\mathbb{Z}, M(C)(1)[2]), \\
C H^{2}\left(C \times \mathbb{P}^{1}, 0\right) & \cong C H^{1}(C, 0) & \oplus & C H^{2}(C, 0),
\end{array}
$$

showing the remaining statement.

\section{Construction of the reciprocity map}

In this section we will construct natural homomorphisms

$$
c_{i}=c_{i, n}: h_{i}(X, \mathbb{Z} / n \mathbb{Z}) \longrightarrow H_{c, e t}^{2 d-i}(X, \mathbb{Z} / n \mathbb{Z}(d))
$$

for any equidimensional scheme $X \in \operatorname{Sm}(k)$ of dimension $d$ and any natural number $n$ prime to $p=\operatorname{char}(k)$. Having done this, we can pass to the inverse limit over all $\ell^{r}, r \geq 0$ ( $\ell$ a prime number $\neq p$ ), and we obtain homomorphisms

$$
h_{0}(X) \rightarrow h_{0}(X) \otimes \mathbb{Z}_{\ell} \rightarrow H_{c, e t}^{2 d}\left(X, \mathbb{Z}_{\ell}(d)\right) .
$$

If the field $k$ is finite, Poincare duality identifies the right hand group with the pro- $\ell$-part $\pi_{1}(X)^{a b}(\ell)$ of the abelianized étale fundamental group of $X$. The composition of these maps defines the prime-to- $p$-part of the reciprocity homomorphism rec of the introduction. We define the $p$-part of rec as the composition map

$$
h_{0}(X) \rightarrow h_{0}(\bar{X}) \cong \mathrm{CH}_{0}(\bar{X}) \stackrel{\alpha}{\rightarrow} \pi_{1}(\bar{X})^{a b}(p) \cong \pi_{1}^{t}(X)^{a b}(p),
$$

where $\alpha$ is the $p$-part of the reciprocity map $\mathrm{CH}_{0}(\bar{X}) \rightarrow \pi_{1}(\bar{X})^{a b}$ defined by Kato and Saito [KS1]. 
This yields a natural homomorphism

$$
r e c: h_{0}(X) \longrightarrow \pi_{1}^{t}(X)^{a b} .
$$

Furthermore, the class $[x]$ of a closed point $x$ of $X$ in $h_{0}(X)$ is sent to the Frobenius of $x$ in the abelianized tame fundamental group. For the $p$-part, this is true by construction. For the prime-to- $p$ part, this follows by considering the case of dimension zero, the morphism $\{x\} \rightarrow X$ and the fact that the maps $c_{i}$ constructed below are natural with respect to scheme morphisms. The commutative diagram

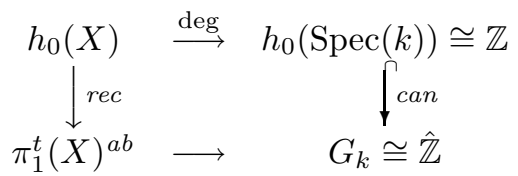

implies the existence of

$$
\operatorname{rec}_{0}: h_{0}(X)^{0} \longrightarrow\left(\pi_{1}^{t}(X)^{a b}\right)^{0} .
$$

Now we come to the construction of the maps $c_{i}$. Consider the complex of sheaves $\mathcal{C}^{\bullet}(X, \mathbb{Z} / n \mathbb{Z})$ on the small étale site on $X$ which is associated to the complex of presheaves

$$
U \longmapsto \operatorname{Hom}\left(C_{\bullet}\left(c_{0}(U)\right), \mathbb{Z} / n \mathbb{Z}\right) .
$$

Consider the homomorphism

$$
\phi: \mathbb{Z} / n \mathbb{Z} \longrightarrow \mathcal{C}^{\bullet}(X, \mathbb{Z} / n \mathbb{Z})
$$

$(\mathbb{Z} / n \mathbb{Z}$ also denotes the constant sheaf associated to $\mathbb{Z} / n \mathbb{Z}$ considered as a complex concentrated in degree zero), which is defined by sending $\overline{1} \in \mathbb{Z} / n \mathbb{Z}$ to the element in $\operatorname{Hom}\left(C_{\bullet}\left(c_{0}(X)\right), \mathbb{Z} / n \mathbb{Z}\right)$ which sends a closed point $P$ on $X$ to the class of $[k(P): k]$ in $\mathbb{Z} / n \mathbb{Z}$. Considering the stalks of $\mathcal{C} \bullet(X, \mathbb{Z} / n \mathbb{Z})$, ([SV1], th.4.3), implies that $\phi$ is a quasiisomorphism. We therefore obtain a natural homomorphism

$$
\operatorname{Hom}\left(C_{\bullet}\left(c_{0}(X)\right), \mathbb{Z} / n \mathbb{Z}\right) \rightarrow \mathbf{R} \Gamma(X, \mathbb{Z} / n \mathbb{Z})=\mathbf{R} \Gamma\left(\operatorname{Spec}(k), \mathbf{R} f_{*}(\mathbb{Z} / n \mathbb{Z})\right),
$$

where $f: X \rightarrow \operatorname{Spec}(k)$ is the structural morphism. Applying the same construction to $X_{K}=X \otimes_{k} K$, where $K$ runs through all finite extensions of $k$, we obtain a homomorphism of complexes of $G_{k}$-modules (or of objects in $\left.\mathrm{D}^{-}\left(\operatorname{Shv}_{\text {ét, } \mathbb{Z} / n \mathbb{Z}}(\operatorname{Spec}(k))\right)\right)$

$$
\operatorname{Hom}\left(C_{\bullet}\left(X_{\bar{k}}\right), \mathbb{Z} / n \mathbb{Z}\right) \longrightarrow \mathbf{R} \bar{f}_{*}(\mathbb{Z} / n \mathbb{Z}) .
$$

By [SV1] it is a quasiisomorphism (see [Ge] for the necessary modifications in positive characteristic using de Jong's theorem on alterations [dJ]). Inverting it 
in $\mathrm{D}^{-}\left(\operatorname{Shv}_{\text {ét, }} \mathbb{Z} / n \mathbb{Z}(\operatorname{Spec}(k))\right)$ and applying étale Poincaré duality for the smooth scheme $X$ therefore gives a natural homomorphism in $\mathrm{D}^{-}\left(\operatorname{Shv}_{\text {ét }, \mathbb{Z} / n \mathbb{Z}}(\operatorname{Spec}(k))\right)$

$$
\underline{C} .\left(c_{0}(X) \otimes \mathbb{Z} / n \mathbb{Z}\right) \longrightarrow \mathbf{R} f_{!}(\mathbb{Z} / n \mathbb{Z})(d)[2 d] .
$$

Applying the functor $\mathbf{R} \Gamma(k,-)$, we thus obtain a natural homomorphism in $\mathrm{D}(\mathbb{Z} / n \mathbb{Z}$-modules $)$

$$
\begin{aligned}
& \left.C \bullet(X, \mathbb{Z} / n \mathbb{Z})=\underline{C} \cdot\left(c_{0}(X) \otimes \mathbb{Z} / n \mathbb{Z}\right)\right)(\operatorname{Spec}(k)) \longrightarrow \\
& \quad \mathbf{R} \Gamma\left(k, \underline{C} \cdot\left(c_{0}(X) \otimes \mathbb{Z} / n \mathbb{Z}\right)\right) \longrightarrow \mathbf{R} \Gamma\left(k, \mathbf{R} f_{!}(\mathbb{Z} / n \mathbb{Z})(d)[2 d]\right) .
\end{aligned}
$$

Finally, taking cohomology, we obtain the required homomorphisms

$$
c_{i, n}: h_{i}(X, \mathbb{Z} / n \mathbb{Z}) \longrightarrow H_{e t, c}^{2 d-i}(X, \mathbb{Z} / n \mathbb{Z}(d)) .
$$

The assignment

$$
K \longmapsto z^{r}\left(X_{K}, \bullet, \mathbb{Z} / n \mathbb{Z}\right)
$$

defines a complex of sheaves $\underline{z}^{r}(X, \bullet, \mathbb{Z} / n \mathbb{Z})$ in $\operatorname{Shv}_{\text {ét, }} \mathbb{Z} / n \mathbb{Z}(\operatorname{Spec}(k))$, and if $X$ is quasiprojective of dimension $d$, then Suslin $[\mathrm{S}]$ (see again [Ge] for the necessary modifications in positive characteristic) shows that the natural inclusion

$$
\underline{C} .\left(z_{0}(X) \otimes \mathbb{Z} / n \mathbb{Z}\right) \longrightarrow \underline{z}^{d}(X, \bullet, \mathbb{Z} / n \mathbb{Z})
$$

induces an isomorphism in $\mathrm{D}^{-}\left(\operatorname{Shv}_{\text {ét, }} \mathbb{Z} / n \mathbb{Z}(\operatorname{Spec}(k))\right)$. Thus, if $X$ is projective, we obtain a commutative diagram

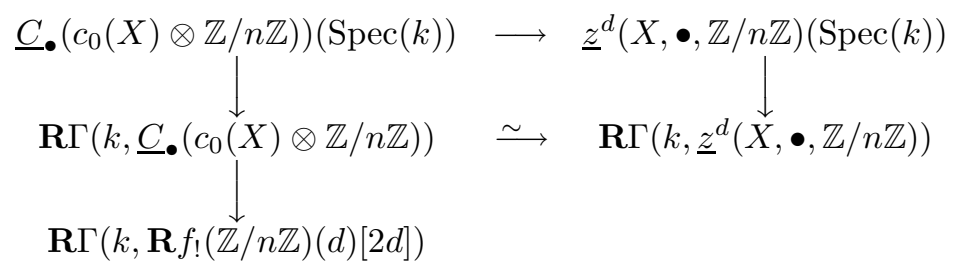

Therefore, if $X$ is smooth and projective, the map

$$
c_{i, n}: h_{i}(X, \mathbb{Z} / n \mathbb{Z}) \longrightarrow H_{e t}^{2 d-i}(X, \mathbb{Z} / n \mathbb{Z}(d))
$$

factors through $\mathrm{CH}^{d}(X, i, \mathbb{Z} / n \mathbb{Z})$. Summarizing, we obtain the

Proposition 3.1 Let $k$ be a perfect field and let $X$ be a smooth, geometrically connected variety of dimension $d$ over $k$. Let $n$ be an integer prime to the characteristic of $k$. Then we have natural homomorphisms for all $i$

$$
c_{i, n}: h_{i}(X, \mathbb{Z} / n \mathbb{Z}) \longrightarrow H_{c, \text { et }}^{2 d-i}(X, \mathbb{Z} / n \mathbb{Z}(d)) .
$$

If $X$ is projective, then $c_{i, n}$ factors through the canonical homomorphism

$$
h_{i}(X, \mathbb{Z} / n \mathbb{Z}) \stackrel{\text { can }}{\longrightarrow} \mathrm{CH}^{d}(X, i, \mathbb{Z} / n \mathbb{Z}) \longrightarrow H_{e t}^{2 d-i}(X, \mathbb{Z} / n \mathbb{Z}(d)) .
$$


Remarks. (a) More generally for $X$ as in prop.3.1 and integers $i, j$ with $i \geq 0$ we have natural maps

$$
\operatorname{Hom}_{\mathrm{DM}_{-}^{\mathrm{eff}}(k)}(\mathbb{Z}, M(X, \mathbb{Z} / n \mathbb{Z})(i)[j]) \longrightarrow H_{e t, c}^{2 d+j}(X, \mathbb{Z} / n \mathbb{Z}(d+i)) .
$$

(b) The morphisms $c_{i}$ are functorial with respect to Gysin sequences. More precisely let $X$ be a smooth variety over a perfect field $k$ and $Z$ a smooth closed subscheme of $X$ everywhere of codimension $c$. Denote by $Y$ the complement of $Z$ in $X$. Then we have a commutative diagram with exact columns

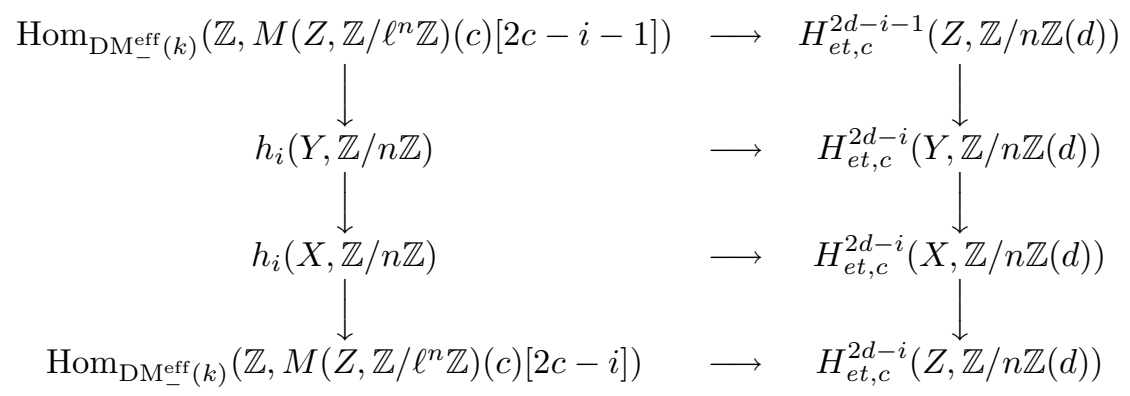

where the first column is induced by the Gysin triangle of $Z \subseteq X$ in $\mathrm{DM}_{-}^{\text {eff }}(k)$ ([V1], prop.3.5.4) and the horizontal maps are the $c_{i}$.

\section{Proof of the main theorem for $\operatorname{dim} X \leq 2$}

In this section we will prove theorem 0.1 for $\operatorname{dim} X \leq 2$. The general case will follow from this by a hypersurface section argument.

Theorem 4.1 Let $k$ be a finite field and let $X$ be smooth, projective and geometrically connected scheme of dimension $d \leq 2$ over $k$. Let $n$ be an integer prime to $p=\operatorname{char}(k)$. Then the natural homomorphism

$$
c_{i, n}: h_{i}(X, \mathbb{Z} / n \mathbb{Z}) \longrightarrow H_{e t}^{2 d-i}(X, \mathbb{Z} / n \mathbb{Z}(d))
$$

is an isomorphism for $i=0,1$.

Proof: By 2.7 and 3.1, it suffices to show that

$$
\mathrm{CH}^{d}(X, i, \mathbb{Z} / n \mathbb{Z}) \longrightarrow H_{e t}^{2 d-i}(X, \mathbb{Z} / n \mathbb{Z}(d))
$$

is an isomorphism for $i=0,1$. For $i=0$, this follows from ([KS1], th.1). For $d=1=i$, it follows from ([B1], th.6.1), together with the Kummer sequence. Let $d=2, i=1$. Consider the Zariski sheaf on $X$

$$
\mathcal{H}^{2}(X, \mathbb{Z} / n \mathbb{Z}(2))
$$


which is associated to the presheaf $U \mapsto H_{e t}^{2}(U, \mathbb{Z} / n \mathbb{Z}(2))$. By ([BO], th.4.2.2), we have a flasque resolution

$$
\begin{aligned}
& 0 \rightarrow \mathcal{H}^{2}(X, \mathbb{Z} / n \mathbb{Z}(2)) \rightarrow \bigoplus_{x \in X^{0}} i_{x *}\left(H^{2}(k(x), \mathbb{Z} / n \mathbb{Z}(2))\right) \rightarrow \\
& \bigoplus_{x \in X^{1}} i_{x *}\left(H^{1}(k(x), \mathbb{Z} / n \mathbb{Z}(1))\right) \rightarrow \bigoplus_{x \in X^{2}} i_{x *}\left(H^{0}(k(x), \mathbb{Z} / n \mathbb{Z})\right) \rightarrow 0 .
\end{aligned}
$$

Comparing this resolution with the Gersten resolution 1.3, the theorem of Merkur'ev-Suslin induces a natural isomorphism

$$
\mathbf{C H}^{2}(X, 2, \mathbb{Z} / n \mathbb{Z}) \stackrel{\sim}{\longrightarrow} \mathcal{H}^{2}(X, \mathbb{Z} / n \mathbb{Z}(2)) .
$$

Since $\mathrm{CH}^{2}(X, 1, \mathbb{Z} / n \mathbb{Z}) \cong H_{\text {Zar }}^{1}\left(X, \mathbf{C H}^{2}(X, 2, \mathbb{Z} / n \mathbb{Z})\right)$ by 1.2 , in order to prove the theorem, it remains to show that

$$
H_{\text {Zar }}^{1}\left(X, \mathcal{H}^{2}(X, \mathbb{Z} / n \mathbb{Z}(2)) \cong H_{\text {et }}^{3}(X, \mathbb{Z} / n \mathbb{Z}(2)) .\right.
$$

This follows from the exact sequence

$$
H_{\text {Zar }}^{1}\left(X, \mathcal{H}^{2}(X, \mathbb{Z} / n \mathbb{Z}(2))\right) \hookrightarrow H_{\text {et }}^{3}(X, \mathbb{Z} / n \mathbb{Z}(2)) \rightarrow H_{\text {Zar }}^{0}\left(X, \mathcal{H}^{3}(X, \mathbb{Z} / n \mathbb{Z}(2))\right),
$$

since the group on the right hand vanishes by ([CSS], $\S 2.4$, Rem.2).

Corollary 4.2 Let $C$ be a smooth, projective curve over $k$. Then there is a canonical isomorphism

$$
\operatorname{Hom}_{\mathrm{DM}_{-}^{\text {eff }}(k)}(\mathbb{Z}, M(C, \mathbb{Z} / n \mathbb{Z})(1)[1]) \stackrel{\sim}{\longrightarrow} H_{e t}^{3}(C, \mathbb{Z} / n \mathbb{Z}(2)) .
$$

Proof: This follows from 4.1 by considering the surface $C \times \mathbb{P}_{k}^{1}$ and the decompositions of $h_{1}\left(C \times \mathbb{P}_{k}^{1}, \mathbb{Z} / n \mathbb{Z}\right)$ and of $H_{e t}^{3}\left(C \times \mathbb{P}_{k}^{1}, \mathbb{Z} / n \mathbb{Z}(2)\right)$ via correspondences in a similar way as in the proof of 2.8 .

Proof of theorem 0.1 for dimension 2: There exists open immersions $X \subset X^{\prime} \subset \bar{X}$ such that $C:=X^{\prime}-X$ is a smooth curve (not necessarily connected) and $Y:=\bar{X}-X^{\prime}$ is an étale $k$-scheme. Let $C^{\prime}$ be a compactification of $C$ over $k$, i.e. $C^{\prime} / k$ is a smooth, projective curve and $C$ is an open dense subscheme of $C^{\prime}$. We denote by $Z$ the complement of $C$ in $C^{\prime}$. By ([V1], prop.3.5.4), we get three exact triangles in $\mathrm{DM}_{-}^{\text {eff }}(k)$

$$
\begin{aligned}
& M(X) \longrightarrow M\left(X^{\prime}\right) \longrightarrow M(C)(1)[2] \longrightarrow M(X)[1] \\
& M(C) \longrightarrow M\left(C^{\prime}\right) \longrightarrow M(Z)(1)[2] \longrightarrow M(C)[1] \\
& M\left(X^{\prime}\right) \longrightarrow M(Y)(2)[4] \longrightarrow M\left(X^{\prime}\right)[1]
\end{aligned}
$$


By 1.1, the triangle (9) induces an isomorphism

$$
h_{0}\left(X^{\prime}\right) \stackrel{\sim}{\longrightarrow} h_{0}(\bar{X}) .
$$

The triangle (8) together with 1.1 induces isomorphisms for $i \geq 1$

$$
\operatorname{Hom}_{\mathrm{DM}_{-}^{\text {eff }}(k)}(\mathbb{Z}, M(C)(1)[i]) \stackrel{\sim}{\longrightarrow} \operatorname{Hom}_{\mathrm{DM}_{-}^{\text {eff }}(k)}\left(\mathbb{Z}, M\left(C^{\prime}\right)(1)[i]\right)
$$

By 2.8 ,

$$
\operatorname{Hom}_{\mathrm{DM}_{-}^{\text {eff }}(k)}\left(\mathbb{Z}, M\left(C^{\prime}\right)(1)[1]\right) \cong \mathrm{CH}^{2}\left(C^{\prime}, 1\right)
$$

is finite of order prime to $p$ and

$$
\operatorname{Hom}_{\mathrm{DM}_{-}^{\text {eff }}(k)}\left(\mathbb{Z}, M\left(C^{\prime}\right)(1)[2]\right)=0 .
$$

Therefore, (7) induces an exact sequence

$$
\operatorname{Hom}_{\mathrm{DM}_{-}^{\text {eff }}(k)}(\mathbb{Z}, M(C)(1)[1]) \longrightarrow h_{0}(X) \longrightarrow h_{0}\left(X^{\prime}\right) \longrightarrow 0 .
$$

Thus the above sequences show that $h_{0}(X)^{0}$ is finite provided that this holds for $h_{0}(\bar{X})^{0}$. But this follows from ([KS1], th.1), since $h_{0}(\bar{X})=\mathrm{CH}_{0}(\bar{X})$. Furthermore, we obtain an isomorphism of the $p$-torsion subgroups

$$
h_{0}(X)\{p\} \stackrel{\sim}{\longrightarrow} h_{0}(\bar{X})\{p\} .
$$

By ([KS1], th.1), we have $h_{0}(\bar{X})\{p\} \cong\left(\pi_{1}(\bar{X})^{a b}\right)^{0}\{p\}$ and, as explained in the introduction, the latter group coincides with $\left(\pi_{1}^{t}(X)^{a b}\right)^{0}\{p\}$. Therefore, in order to conclude the proof of theorem 0.1 for dimension 2 , it suffices to show that for all $n \geq 1$ and every prime number $\ell \neq p$, the homomorphism

$$
c_{0, \ell^{n}}: h_{0}\left(X, \mathbb{Z} / \ell^{n} \mathbb{Z}\right) \longrightarrow H_{e t, c}^{4}\left(X, \mathbb{Z} / \ell^{n} \mathbb{Z}(2)\right)
$$

is an isomorphism. Indeed, the finiteness of $h_{0}(X)^{0}$ and (11) imply that

$$
\begin{aligned}
& h_{0}(X)^{0}\{\ell\} \cong h_{0}(X)^{0} \otimes \mathbb{Z}_{\ell} \\
& =\operatorname{ker}\left(h_{0}(X) \otimes \mathbb{Z}_{\ell} \stackrel{\operatorname{deg}}{\longrightarrow} \mathbb{Z}_{\ell}\right) \\
& =\operatorname{ker}\left(\lim _{n} h_{0}\left(X, \mathbb{Z} / \ell^{n} \mathbb{Z}\right) \longrightarrow \mathbb{Z}_{\ell}\right) \\
& \cong \operatorname{ker}\left(\lim _{n} H_{e t, c}^{4}\left(X, \mathbb{Z} / \ell^{n} \mathbb{Z}(2)\right) \longrightarrow \mathbb{Z}_{\ell}\right) \\
& =\operatorname{ker}\left(H_{e t, c}^{2 d}\left(X, \mathbb{Z}_{\ell}(d)\right) \rightarrow H_{c}^{2 d}\left(X_{\bar{k}}, \mathbb{Z}_{\ell}(d)\right) \cong \mathbb{Z}_{\ell}\right) \\
& \cong\left(\pi_{1}^{t}(X)^{a b}\right)^{0} \otimes \mathbb{Z}_{\ell} \text {. }
\end{aligned}
$$


Consider the commutative diagrams with exact columns

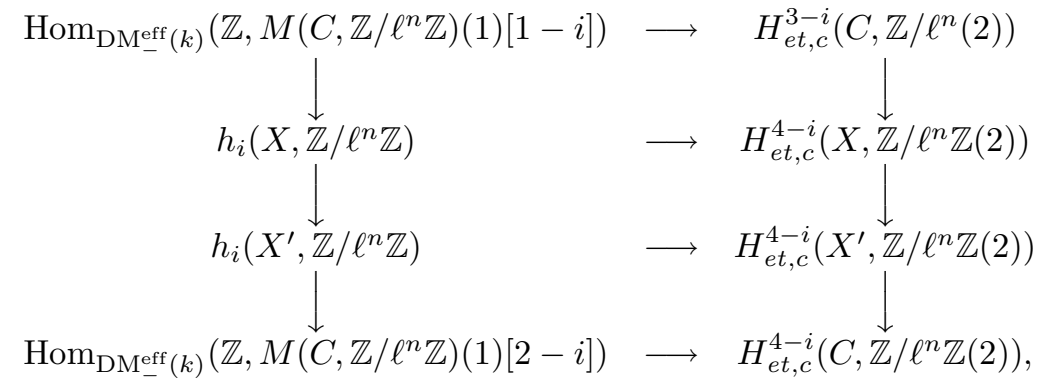

(13)

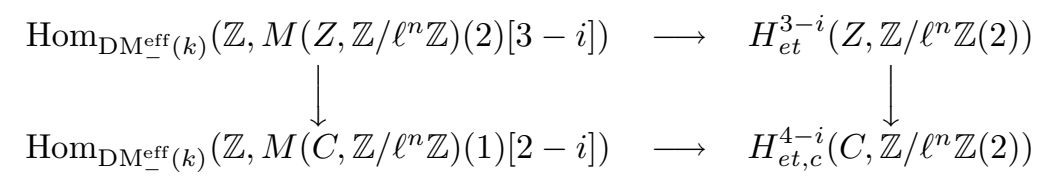

(14)
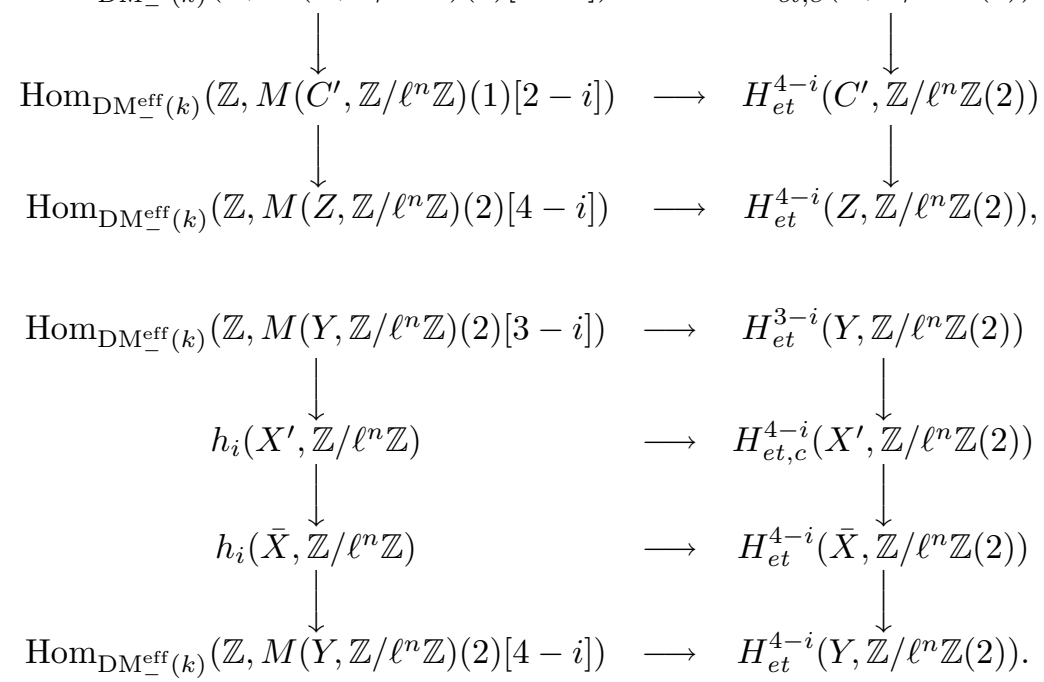

Since the groups in the top and bottom row of diagram (14) vanish for $i=0,1$ we deduce from 4.1 that

$$
c_{i, \ell^{n}}: h_{i}\left(X^{\prime}, \mathbb{Z} / \ell^{n} \mathbb{Z}\right) \longrightarrow H_{e t, c}^{4-i}\left(X^{\prime}, \mathbb{Z} / \ell^{n} \mathbb{Z}(2)\right)
$$

is an isomorphism for $i=0,1$. Moreover, by 4.2 and 2.8, the map

$$
\operatorname{Hom}_{\mathrm{DM}_{-}^{\text {eff }}(k)}\left(\mathbb{Z}, M\left(C^{\prime}, \mathbb{Z} / \ell^{n} \mathbb{Z}\right)(1)[2-i]\right) \longrightarrow H_{e t}^{4-i}\left(C^{\prime}, \mathbb{Z} / \ell^{n}(2)\right)
$$

is an isomorphism for $i=0,1$. Since $\operatorname{Hom}_{\mathrm{DM}_{-}^{\text {eff }}(k)}\left(\mathbb{Z}, M\left(Z, \mathbb{Z} / \ell^{n} \mathbb{Z}\right)(2)[i+1]\right)=$ $0=H_{e t}^{3-i}\left(Z, \mathbb{Z} / \ell^{n} \mathbb{Z}(2)\right)$ for $i=0,1$, diagram (13), 2.8 and (4.2) show that

$$
\operatorname{Hom}_{\mathrm{DM}_{-}^{\text {eff }}(k)}\left(\mathbb{Z}, M\left(C, \mathbb{Z} / \ell^{n} \mathbb{Z}\right)(1)[i]\right) \longrightarrow H_{\text {et }}^{4-i}\left(C, \mathbb{Z} / \ell^{n} \mathbb{Z}(2)\right)
$$

is an isomorphism for $i=0,1$, as well. Finally, diagram (12) and the five-lemma imply that (11) is bijective. 


\section{Proof in the general case}

In order to extend the result from dimension $\leq 2$ to arbitrary dimensions, we are going to use a hypersurface section argument similar to that proposed by Colliot-Thélène in the projective case (see [KS1]). Unfortunately, the following Bertini-type theorem does not follow directly from ([AK], th.1 and th.7); one has modify the arguments given there.

Theorem 5.1 Let $X \subset \mathbb{P}=\mathbb{P}(W)$ be a smooth, projective, geometrically connected variety of dimension $\geq 3$ over an infinite field $k$. Let $Z_{1}, \ldots, Z_{s}$, $Z_{s+1}, \ldots, Z_{t}$ be geometrically irreducible subvarieties in $X$, where $Z_{1}, \ldots, Z_{s}$ are of codimension 1 in $X$. Furthermore, let $P_{1}, \ldots, P_{r}$ be closed points in $X-\left(\cup_{i=1}^{t} Z_{i}\right)$. Let $I$ be the ideal defining $\left\{P_{1}, \ldots, P_{r}\right\}$ in $\mathbb{P}$ and let $n$ be chosen such $I(n)$ is generated by global sections. Let $H \subset \mathbb{P}$ be a generic hypersurface of degree $n+1$ containing $P_{1}, \ldots, P_{r}$. Then $H \cap X$ is smooth and geometrically connected, $H$ does not contain $Z_{i}, i=1, \ldots, t$ and $H \cap Z_{i}$ is geometrically irreducible of codimension 1 in $Z_{i}$ for $i=1, \ldots, s$.

Sketch of proof following [AK]: Let $Y=X-\left\{P_{1}, \ldots, P_{r}\right\}$. The $\mathcal{O}_{Y}$-module $I(n) \mid Y$ is invertible and generated by global sections, so we obtain a morphism

$$
Y \longrightarrow \mathbb{P}(\Gamma(\mathbb{P}, I(n))) \text {. }
$$

Combining it with inclusion $Y \stackrel{i}{\longrightarrow} \mathbb{P}(W)$ and using the Segre embedding, we obtain a composite embedding

$$
Y \longrightarrow \mathbb{P}(\Gamma(\mathbb{P}, I(n))) \times \mathbb{P}(W) \longrightarrow \mathbb{P}(\Gamma(\mathbb{P}, I(n)) \otimes W) .
$$

It is defined by the natural surjection

$$
(\Gamma(\mathbb{P}, I(n)) \otimes W)_{Y} \longrightarrow I(n+1) \mid Y,
$$

which factors through the natural map

$$
(\Gamma(\mathbb{P}, I(n+1)))_{Y} \longrightarrow I(n+1) \mid Y .
$$

Therefore, also the latter map is surjective and we obtain a commutative diagram

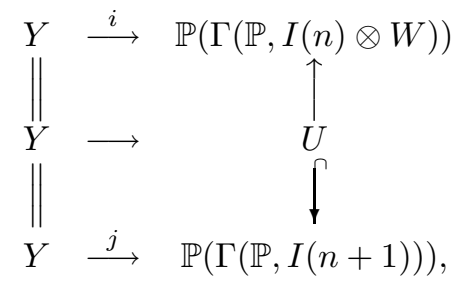

where $U \subset \mathbb{P}(\Gamma(\mathbb{P}, I(n+1)))$ is the open subset on which the morphism $\mathbb{P}(\Gamma(\mathbb{P}, I(n+1))) \rightarrow \mathbb{P}(\Gamma(\mathbb{P}, I(n)) \otimes W)$ is defined. Since $i$ is an embedding, 
$j$ is an embedding. Let $s$ be a general element of $\Gamma(\mathbb{P}, I(n+1))$. It defines via $j$ a section $Y_{1}$ of $Y$ by a general hyperplane of $\mathbb{P}(\Gamma(\mathbb{P}, I(n+1))$. Clearly, $Y_{1}$ does not contain $Z_{1}, \ldots, Z_{t}$ and, using the classical Bertini theorems (see [L1], chap.VII, th.7, prop.13) as in the proof of ([AK], th.1), we see that $Y_{1}$ is smooth and geometrically connected and $Y_{1} \cap Z_{i}$ is geometrically irreducible for $i=1, \ldots, s$. Viewing $s$ as a section of $\mathcal{O}_{\mathbb{P}}(n+1)$, we obtain a general hypersurface section $X_{1}=H \cap X$ which contains $P_{1}, \ldots, P_{r}$ and with

$$
Y_{1}=X_{1} \cap Y \text {. }
$$

We conclude that $X_{1}$ is geometrically irreducible and smooth off $\left\{P_{1}, \ldots, P_{r}\right\}$. Furthermore, $X_{1} \cap Z_{i}=Y_{1} \cap Z_{i}$ for $i=1$,..t. It remains to show that $X_{1}$ is smooth at $P_{1}, \ldots, P_{r}$. This follows from ([AK], th.7).

Corollary 5.2 Let $\bar{X} \subset \mathbb{P}_{k}^{N}$ be a smooth, projective, geometrically connected variety of dimension $\geq 3$ over an infinite perfect field $k$ and let $X \subset \bar{X}$ be an open subscheme. Let finitely many closed points $P_{1}, \ldots, P_{r} \in X$ be given. Let $Z_{1}, \ldots, Z_{s}$ be the irreducible components of $(\bar{X}-X)_{\bar{k}}$ which are of codimension 1 in $\bar{X}_{\bar{k}}(\bar{k}$ denotes an algebraic closure of $k)$. Then there exists a closed subset $T \subset \bar{X}-X$ of codimension $\geq 2$ in $\bar{X}$ and a hypersurface $H \subseteq \mathbb{P}_{k}^{N}$ containing $P_{1}, \ldots, P_{r}$ such that

(i) $H \cap \bar{X}$ is smooth and geometrically connected,

(ii) $Y_{i}:=Z_{i}-\left(T_{\bar{k}} \cap Z_{i}\right)$ is smooth and connected for $i=1, \ldots, s$,

(iii) $H_{\bar{k}} \cap Y_{i}$ is smooth and connected for $i=1, \ldots, s$,

(iv) $H \cap T$ is of codimension $\geq 2$ in $H \cap \bar{X}$,

(v) $T$ contains every irreducible component of $\bar{X}-X$ which is of codimension $\geq 2$ in $\bar{X}$.

Proof: By 5.1, a generic hypersurface section of sufficiently high degree in $\mathbb{P}_{\bar{k}}^{N}$ containing $\left\{P_{1}, \ldots, P_{r}\right\}_{\bar{k}}$ has an irreducible intersection with $Z_{i}, i=1, \ldots, s$, does not contain any irreducible component of the singular locus of one of the $Z_{i}$ and does not contain any irreducible component of $(\bar{X}-X)_{\bar{k}}$. Since $k$ is infinite, we may choose $H$ defined over $k$ and again by $5.1, H \cap \bar{X}$ is smooth. Now choose $T$ such that $T_{\bar{k}}$ contains all singularities of $Z_{i}$, all singularities of $H_{\bar{k}} \cap Z_{i}, i=1, \ldots, s$ and every irreducible component of $(\bar{X}-X)_{\bar{k}}$ which is of codimension $\geq 2$ in $\bar{X}_{\bar{k}}$.

We use the above result in order to show the

Theorem 5.3 Let $\bar{X} \subset \mathbb{P}_{k}^{N}$ be a smooth, projective, geometrically connected variety of dimension $\geq 3$ over a finite field $k$ and let $X \subset \bar{X}$ be an open subscheme. Let finitely many closed points $P_{1}, \ldots, P_{r} \in X$ be given and let $M$ be a 
positive integer. Then, after replacing $k$ by a finite extension of degree prime to $M$, there exists a hypersurface $H \subset \mathbb{P}_{k}^{N}$ containing $P_{1}, \ldots, P_{r}$ such that $\bar{X} \cap H$ is smooth and geometrically connected and such that the natural homomorphism

$$
\pi_{1}^{t}(X \cap H)^{a b} \longrightarrow \pi_{1}^{t}(X)^{a b}
$$

is an isomorphism.

Proof: Let $Z_{1}, \ldots, Z_{s}$ be the irreducible components of $(\bar{X}-X)_{\bar{k}}$ of codimension 1 in $\bar{X}$. Over any infinite extension of $k$, we find a hypersurface $H$ and a closed subscheme $T \subset \bar{X}-X$ of codimension $\geq 2$ in $\bar{X}$ satisfying the conditions of 5.2. Replacing $k$ by a finite extension, which we may choose of degree prime to $M$, we may assume that $H$ and $T$ are already defined over $k$. Let $X_{1}=\bar{X}-T$. We set $Y_{i}=Z_{i} \cap\left(X_{1}\right)_{\bar{k}}$. Then the following holds.

(i) $\bar{X}-X_{1}$ is of codimension $\geq 2$ in $\bar{X}$,

(ii) $\left(X_{1}-X\right)_{\bar{k}}=Y_{1} \cup \cdots \cup Y_{s}$,

(iii) $\bar{X} \cap H$ is smooth and geometrically connected,

(iv) $Y_{i} \cap H_{\bar{k}}$ is smooth, connected and of codimension 1 in $Y_{i}$ for $i=1, \ldots, s$,

(v) $\bar{X}-(H \cap \bar{X})$ is affine,

(vi) $H \cap T$ is of codimension $\geq 2$ in $H \cap \bar{X}$.

Recall that the étale cohomological dimension of a scheme $U$ of finite type over a finite field is less or equal to $2 \operatorname{dim}(U)+1$ (cf. [Mi], VI, cor.1.4) and is equal to $\operatorname{dim}(U)+1$ if $U$ is affine ([Mi], VI, th.7.2).

Let $n$ be any integer prime to the characteristic $p$ of $k$. By (i), we have

$$
H_{e t}^{2 d-i}\left(\bar{X}-X_{1}, \mathbb{Z} / n \mathbb{Z}(d)\right)=0 \quad \text { for } i<3 .
$$

Thus the exact sequences

$$
\begin{array}{r}
H_{e t}^{2 d-i-1}\left(\bar{X}-X_{1}, \mathbb{Z} / n \mathbb{Z}(d)\right) \rightarrow H_{e t, c}^{2 d-i}\left(X_{1}, \mathbb{Z} / n \mathbb{Z}(d)\right) \stackrel{\alpha_{i}}{\rightarrow} H_{e t}^{2 d-i}(\bar{X}, \mathbb{Z} / n \mathbb{Z}(d)) \\
\rightarrow H_{e t}^{2 d-i}\left(\bar{X}-X_{1}, \mathbb{Z} / n \mathbb{Z}(d)\right)
\end{array}
$$

show that $\alpha_{i}$ is an isomorphism for $i=0,1$. The same reasoning applied to $H \cap\left(\bar{X}-X_{1}\right)$ shows (using property (vi) above) that

$$
H_{e t, c}^{2 d-2-i}\left(X_{1} \cap H, \mathbb{Z} / n \mathbb{Z}(d-1)\right) \stackrel{\beta_{i}}{\longrightarrow} H_{e t}^{2 d-2-i}(\bar{X} \cap H, \mathbb{Z} / n \mathbb{Z}(d-1))
$$

is an isomorphism for $i=0,1$. Now consider the following commutative diagram for $i=0,1$ 


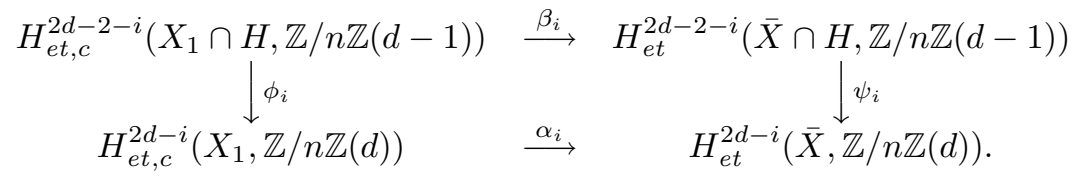

The arrow $\psi_{i}$ is induced via Poincaré duality from the natural homomorphism $H_{e t}^{1}(\bar{X}, \mathbb{Z} / n \mathbb{Z}) \rightarrow H_{e t}^{1}(\bar{X} \cap H, \mathbb{Z} / n \mathbb{Z})$ and the definition of $\phi$ is similar.

Since $\bar{X}-(H \cap \bar{X})$ is affine, we have $H_{e t}^{i}(\bar{X}-(H \cap \bar{X}), \mathbb{Z} / n \mathbb{Z}(d))=0$ for $i>d+1$. Hence the Gysin sequence ([Mi],VI,5.4) shows that $\psi_{i}$ is an isomorphism for $i=0$ and is surjective for $i=1$. Therefore, the same is true for $\phi_{i}$.

Now put $D=X_{1}-X$, thus $D_{\bar{k}}=Y_{1} \cup \cdots \cup Y_{s}$. We consider the following commutative diagram with exact columns

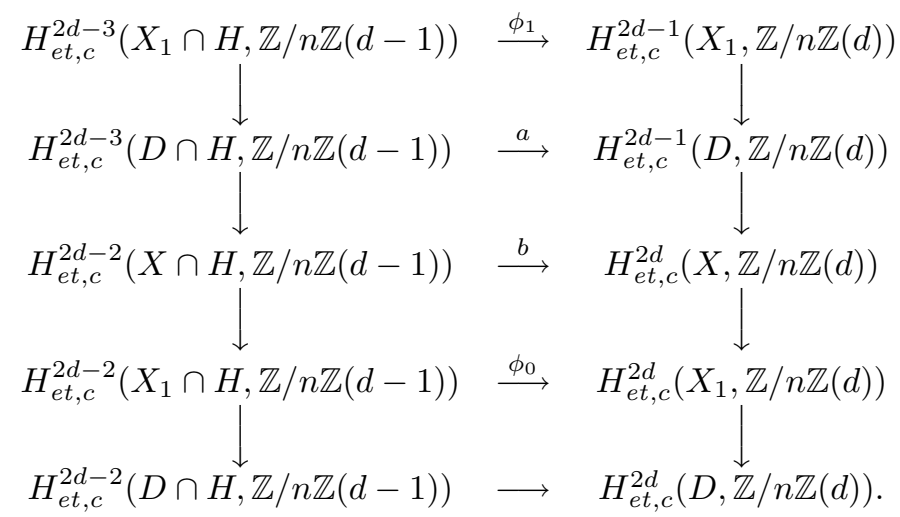

Both objects on the lower line are zero by dimension reasons, $\phi_{0}$ is an isomorphism and $\phi_{1}$ is surjective. Thus, in order to show that $b$ is a isomorphism, it suffices to show that $a$ is an isomorphism. Since $G_{k} \cong \hat{\mathbb{Z}}$, the Hochschild-Serre spectral sequence induces isomorphisms

$$
H_{e t, c}^{2 d-3}(D \cap H, \mathbb{Z} / n \mathbb{Z}(d-1)) \cong H^{1}\left(G_{k}, H_{e t, c}^{2 d-4}\left((D \cap H)_{\bar{k}}, \mathbb{Z} / n \mathbb{Z}(d-1)\right)\right)
$$

and

$$
H_{e t, c}^{2 d-1}(D, \mathbb{Z} / n \mathbb{Z}(d)) \cong H^{1}\left(G_{k}, H_{e t, c}^{2 d-2}\left(D_{\bar{k}}, \mathbb{Z} / n \mathbb{Z}(d)\right)\right) .
$$

Therefore, it suffices to show that $a \otimes \bar{k}$ is an isomorphism. This, however follows via Poincaré duality using property (iv) above. We have shown that the natural homomorphism

$$
H_{e t, c}^{2 d-2}(X \cap H, \mathbb{Z} / n \mathbb{Z}(d-1)) \longrightarrow H_{e t, c}^{2 d}(X, \mathbb{Z} / n \mathbb{Z}(d))
$$

is an isomorphism. Another application of Poincaré duality therefore implies that the natural map

$$
\pi_{1}(X \cap H)^{a b} / n \longrightarrow \pi_{1}(X)^{a b} / n
$$


is an isomorphism. Since $n$ was arbitrary prime to $p$, this shows the statement of the theorem for the prime-to- $p$-part. The statement on the $p$-part follows from the commutative diagram

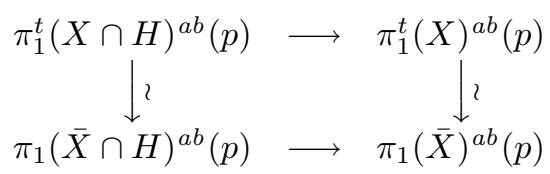

and from the fact that the lower horizontal arrow is an isomorphism by ([SGA2], Exp.XII, Cor.3.5).

Now we complete the proof of theorem 0.1. We proceed by induction on the dimension of $X$. For dimension $\leq 2$ everything is proved, so assume that $\operatorname{dim} X=d \geq 3$ and that the theorem is true in dimensions $<d$. Choose a hypersurface section $H$ according to 5.3 (with any points $P_{1}, \ldots, P_{r}$ ). We obtain a commutative diagram

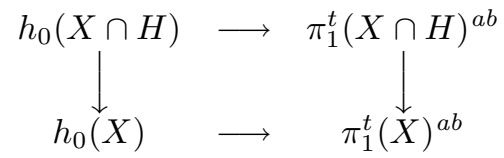

which exists over a finite extension of $k$ whose degree can be chosen prime to any given integer $M$. Using the usual restriction-corestriction argument, the induction hypothesis and theorem 5.3 imply that

$$
\operatorname{rec}_{0}:\left(h_{0}(X)\right)^{0} \rightarrow\left(\pi_{1}^{t}(X)^{a b}\right)^{0}
$$

is surjective and that $\left(\pi_{1}^{t}(X)^{a b}\right)^{0}$ is finite. Now let $z$ be a cycle representing an element in the kernel of $r e c_{0}$. Then, over a finite extension of $k$ whose degree can be chosen prime to any given integer $M$, there exists a hypersurface section $H$ according to 5.3 containing the support of $z$. The same reasoning as above shows that the class $[z]$ of $z$ in $h_{0}(X)$ is annihilated by the degree of the finite field extension, hence $r e c_{0}$ is injective.

\section{References}

[AK] A. Altman, S. Kleiman Bertini theorems for hypersurface sections containing a subscheme. Comm. Alg. 7 (1979), 775-790

[B1] S. Bloch Algebraic cycles and higher K-theory. Adv. in Math. 61 (1986), 267-304.

[B2] S. Bloch, The Moving Lemma for Higher Chow Groups. Journal of Algebraic Geometry 3 (1994), 537-568 
[BO] S. Bloch, A. Ogus Gersten's conjecture and the homology of schemes. Ann. Sci. École Norm. Sup. 7 (1974), 181-202.

[CSS] J.-L. Colliot-Thélène, J.-J. Sansuc, C. Soulé Torsion dans le groupe de Chow de codimension deux. Duke Math. J. 50 (1983), 763-801.

[FV] E. Friedlander, V. Voevodsky Bivariant cycle cohomology. In V. Voevodsky, A. Suslin, E. M. Friedlander: Cycles, Transfers, and Motivic Homology Theories. Annals of Math. Studies 143, Princeton University Press 1999

[Ge] Th. Geisser Applications of de Jong's theorem on alterations. In H.Hauser, J.Lipman, F.Oort, A.Quir'os (eds.): Resolution of Singularities. In tribute of Oscar Zariski. Progress in Math., Birkhäuser 1999

[GM] A. Grothendieck, J. P. Murre The tame fundamental group of a formal neighbourhood of a divisor with normal crossings on a scheme. Springer Lect. Notes Math. 208 (1971)

[dJ] A. J. de Jong Smoothness, semi-stability and alterations. Publ. Math. IHES 83 (1996), 51-93.

[KS1] K. Kato, S. Saito, Unramified class field theory of arithmetical surfaces. Ann. of Math. 118 (1983), 241-275.

[KS2] K. Kato, S. Saito Global class field theory of arithmetic schemes. In S. Bloch, R.K. Dennis, E. M. Friedlander, M. Stein (eds.): Applications of algebraic K-theory to algebraic geometry and number theory. Contemp. Math. vol. 55 (1986), Amer. Math. Soc., Providence RI, $255-331$.

[L1] S. Lang Introduction to algebraic geometry. Interscience, New York 1958

[L2] S. Lang Sur le séries L d'une variété algébrique. Bull. Soc. Math. France 84 (1956), 555-563.

[Li] J. Lipman Desingularization of two-dimensional schemes. Ann. o. Math. 107 (1978), 151-207.

[Mi] J. Milne Étale cohomology. Princeton University Press 1980

[Mo] C. Moore Group extensions of p-adic and adelic linear groups. Publ. Math. I.H.E.S. 35 (1969), 5-74.

[NS] Yu. P. Nesterenko, A. A. Suslin Homology of the full linear group over a local ring, and Milnor's K-theory (in Russian). Izv. Akad. Nauk SSSR 53 (1989), 121-146; Engl. Transl.: Math. USSR Isv. 34 (1990), $121-145$. 
[Ra] W. Raskind Abelian Class Field Theory of Arithmetic Schemes. Proceedings of Symposia in Pure Math. 58.1 (1995), 85-187.

[Sa] S. Saito Unramified class field theory of arithmetical schemes. Ann. o. Math. 121 (1985), 251-281.

[Sch] A. Schmidt Absolute Singular Homology of Arithmetic Schemes. In preparation

[S] A. Suslin Higher Chow groups and étale cohomology, Preprint, 1994

[SV1] A. Suslin, V. Voevodsky Singular homology of abstract algebraic varieties. Invent. Math. 123 (1996), 61-94.

[SV2] A. Suslin, V. Voevodsky Relative cycles and Chow sheaves. In V. Voevodsky, A. Suslin, E. M. Friedlander: Cycles, Transfers, and Motivic Homology Theories. Annals of Math. Studies 143, Princeton University Press 1999

[SV3] A. Suslin, V. Voevodsky Bloch-Kato conjecture and motivic cohomology with finite coefficients (new version). Preprint 1999

[To] B. Totaro Milnor K-theory is the simplest part of algebraic K-theory. $K$-Theory 6 (1990), 177-189.

[V1] V. Voevodsky Triangulated categories of motives over a field. In V. Voevodsky, A. Suslin, E. M. Friedlander: Cycles, Transfers, and Motivic Homology Theories. Annals of Math. Studies 143, Princeton University Press 1999

[V2] V. Voevodsky Cohomological theory of presheaves with transfer. In V. Voevodsky, A. Suslin, E. M. Friedlander: Cycles, Transfers, and Motivic Homology Theories. Annals of Math. Studies 143, Princeton University Press 1999

[SGA1] A. Grothendieck Revètements Étales and Group de Fondamental. Springer Lect. Notes Math. 224 (1971)

[SGA2] A. Grothendieck Cohomologie locale des faisceaux cohérent et théorèmes de Lefschetz locaux et globaux. Masson et Cie, North Holland, Amsterdam 1968
Alexander Schmidt
Michael Spieß
Mathematisches Institut
School of Mathematical Sciences
der Universität Heidelberg
University of Nottingham
Im Neuenheimer Feld 288
University Park
69120 Heidelberg
Germany
NG7 2RD Nottingham
United Kingdom
e-mail: schmidt@mathi.uni-heidelberg.de
mks@maths.nott.ac.uk 